\title{
Robust Grid Adaptation for Efficient Uncertainty
}

\section{Quantification}

\author{
Francisco Palacios ${ }^{1}$ and Karthik Duraisamy ${ }^{2}$ and Juan J. Alonso ${ }^{3}$ \\ Stanford University, Stanford CA 94305, USA \\ Enrique Zuazua ${ }^{4}$ \\ Basque Center for Applied Mathematics, Derio 48160, Spain \\ Ikerbasque - Basque Foundation for Science, Bilbao 48011, Spain
}

In the recent past, adjoint methods have been successfully applied in error estimation of integral outputs (functionals) of the numerical solution of partial differential equations. The adjoint solution can also be used as a grid adaptation indicator, with the objective of optimally targeting and reducing the numerical error in the functional of interest below a pre-specified threshold. In situations where we seek to quantify the effect of aleatory uncertainties on statistical moments of the output functional, it becomes necessary to evaluate the functional accurately at multiple sample points in probability space. If the numerical accuracy of these sample evaluations is not uniform, variations in the numerical error can affect the evaluation of the statistical moments. While it is possible to independently adapt the meshes for the solution at each sample point in stochastic space, such a procedure can be both cumbersome and computationally expensive. To improve on the efficiency of this process, we propose a new robust grid adaptation technique that is aimed at minimizing the numerical error over a range of variations of the uncertain parameters of interest about a nominal state. Using this approach, we are able to generate computational grids that are insensitive to small

\footnotetext{
${ }^{1}$ Engineering Research Associate, Department of Aeronautics and Astronautics, AIAA Member.

${ }^{2}$ Consulting Assistant Professor, Department of Aeronautics and Astronautics, AIAA Member.

3 Associate Professor, Department of Aeronautics and Astronautics, AIAA Senior Member.

${ }^{4}$ Ikerbasque Research Professor \& Scientific Director.
} 
variations of the uncertain parameters that can both locally and globally change the solution and, as a result, the error distribution. This is in contrast with classical adjoint techniques which seek to adapt the grid with the aim of minimizing numerical errors for a specific flow condition (and geometry). We demonstrate that flow computations on these robust grids result in low numerical errors under the expected range of variations of the uncertain input parameters. The effectiveness of this strategy is demonstrated in problems involving the Poisson equation and the Euler equations at transonic and supersonic/hypersonic speeds.

\section{Nomenclature}

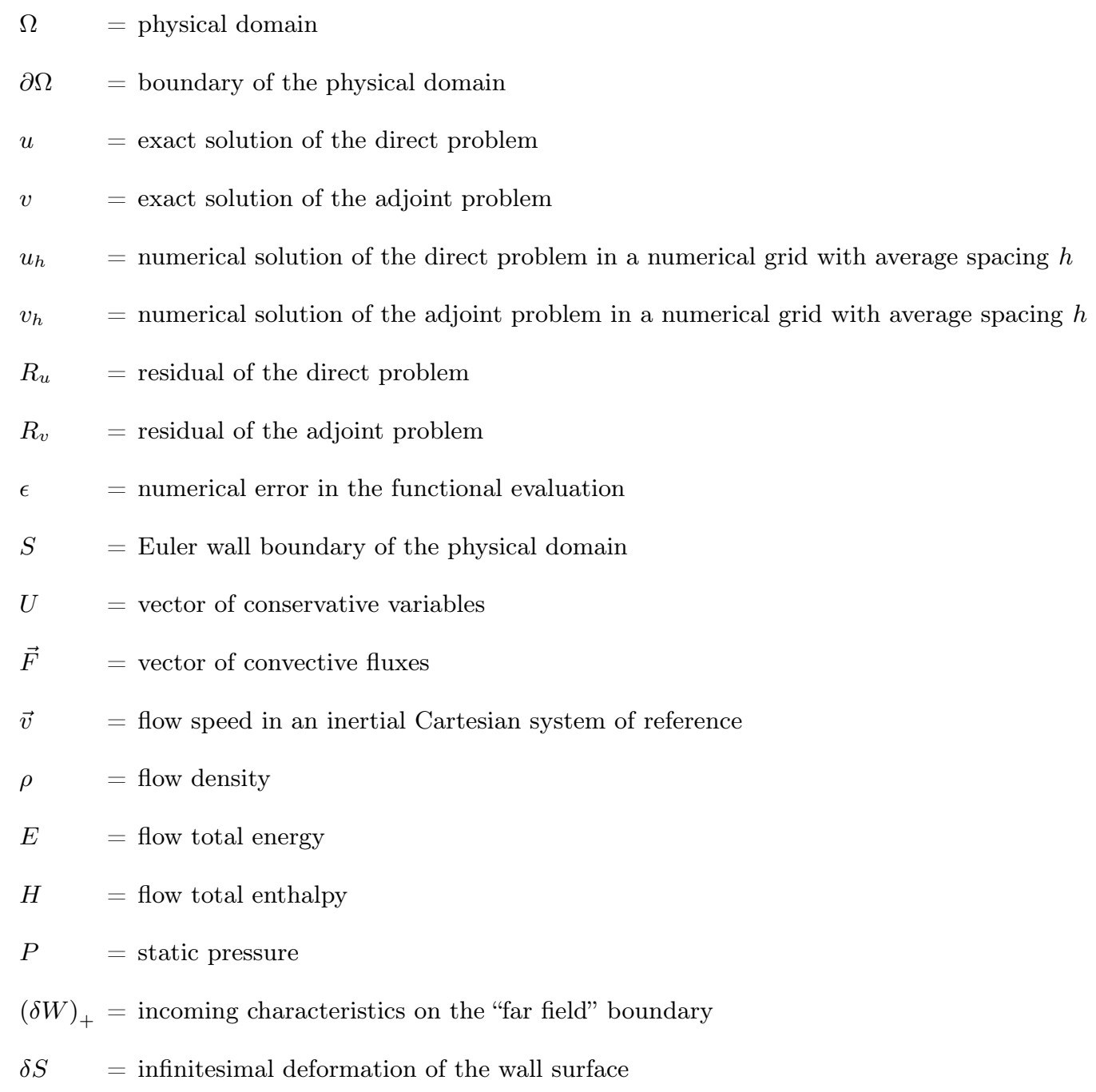




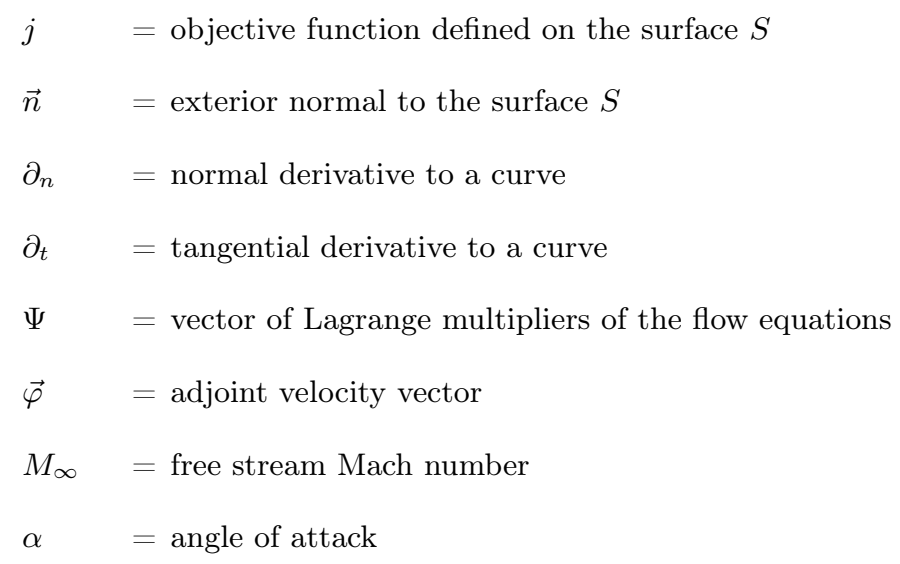

\section{Introduction}

An important aspect of uncertainty quantification in predictive simulations involves the propagation of the effect of variability in input and system parameters (hereafter referred to as input parameters or random input parameters) on system outputs of interest, where we assume that the variability in the input parameters can be represented by known probability distributions. In such exercises, one is typically interested in the statistical moments of an output functional within the space of random input parameters. The non-intrusive approach [1-3] to uncertainty quantification involves sampling of the governing equations in stochastic space. Because of variations in the input parameters and the resulting changes in the magnitude, distribution, and character of the solution of the governing equations, the numerical accuracy of these sample evaluations is not uniform and significant errors could appear in the evaluation of the statistical moments of the outputs. An obvious strategy for coping with this problem would be to apply adjoint-based error estimation and mesh adaptation at every sample point [4-10] so that the numerical errors for all functional evaluations are kept below an acceptable threshold. However, such a procedure can be both cumbersome and computationally expensive. New approaches that can retain the accuracy of such an approach but with much lower computational effort are sought in this paper.

In this work, we propose a robust grid adaptation technique which is aimed at minimizing the numerical discretization error over small variations of the input parameters around a baseline flow state. In this approach, computational grids are generated with the knowledge that small variations 
of uncertain parameters (or design variables) can locally and globally change the solution (and hence, the error distribution).

This grid adaptation strategy is expected to be useful in many applications including uncertainty quantification, robust design, and also in response surface generation with applications in multiple fields of computational science. In all of these situations, it is desirable to have a fixed computational grid which produces low numerical errors when the input parameters of the problem (e.g. shape of the airplane, Mach number, angle of attack, coefficients of different physical models, etc.) change by a small amount. It will be shown that this robust grid adaptation technique is closely related to ideas of error estimation in integral outputs (functionals) of partial differential equations but, instead, the objective in this work is to minimize the variation of the numerical error when an infinitesimal change over the nominal conditions takes place. The first obvious benefit is that an adapted mesh is only constructed once and can be reused, without modification, over large numbers of sample function evaluations, thus decreasing the computational costs. Moreover, removing the requirement of independent mesh adaptation for each sample function evaluation increases the robustness of the procedure, particularly when these sample evaluations constitute an inner loop for other processes such as optimization (in both robust design and reliability-based design) and in other nested uncertainty quantification approaches based on the generation of response surfaces in stochastic space [11].

\section{Robust grid adaptation methodology}

\section{A. Foundations of the methodology}

It is well known that the error estimate of integral outputs of partial differential equations can be used as a grid adaptation indicator $[5-7,9,10]$. These techniques produce good (and even optimal) numerical grids for the accurate estimation of an output functional. However, in principle, this strategy is not well suited for situations in which certain parameters of the baseline configuration change while the grid remains unchanged. In such a scenario, a robust grid adaptation is desirable.

In order to illustrate the main idea behind this technique, assume a system governed by a linear 
partial differential equation [12]. Let $u$ be the solution of the equation:

$$
\left\{\begin{array}{l}
A u=f, \text { in } \Omega, \\
u=0, \quad \text { on } \partial \Omega,
\end{array}\right.
$$

where, for purposes of demonstration, the domain $\Omega$ is subject to homogeneous boundary conditions for which the problem is well-posed. The dual or adjoint problem can be introduced as

$$
\begin{cases}A^{*} v=g, & \text { in } \Omega, \\ v=0, & \text { on } \partial \Omega,\end{cases}
$$

and, due to the duality property, it is possible to verify that

$$
(v, A u)=\left(A^{*} v, u\right)
$$

where (...) represents the scalar inner product, i.e. $(v, u)=\int_{\Omega} v^{T} u d V$.

We are interested in functionals of the solution $u, L_{g}(u)$, and, for purposes of this discussion, a linear function of the state $u$ will be considered. The weight or density function $g$ that defines our functional of interest is given by

$$
L_{g} u=(g, u)=(v, f)=L_{f} v
$$

where $u$ satisfies the primal Eq. (1) and $v$ satisfies the dual or adjoint equation Eq. (2). Next, we introduce a standard Finite Element Method (FEM) approximation where $u_{h}$ and $v_{h}(h$ is meant to represent an average mesh-size measure) are discrete approximations to $u$ and $v$ respectively. The objective is to find a suitable expression for the numerical error $\epsilon=\left(g, u-u_{h}\right)$ in the functional evaluation. The standard procedure $[4,12]$ is as follows:

$$
\begin{aligned}
\epsilon & =\left(g, u-u_{h}\right)=\left(A^{*} v, u-u_{h}\right) \\
& =\left(A^{*} v_{h}, u-u_{h}\right)-\left(A^{*}\left(v_{h}-v\right), u-u_{h}\right) \\
& =\left(v_{h}, A\left(u-u_{h}\right)\right)-\left(v_{h}-v, A\left(u-u_{h}\right)\right) .
\end{aligned}
$$

We introduce the discrete flow and adjoint equation residuals $R_{u} \equiv A u_{h}-f$ and $R_{v} \equiv A^{*} v_{h}-g$. Note that the residual is a computable indicator of the extent to which $u_{h}$ and $v_{h}$ depart from the exact solutions on the numerical grid characterized by $h$. The final expression for the error estimate 


$$
\epsilon=\left(v_{h}, R_{u}\right)-\left(v_{h}-v, A\left(u-u_{h}\right)\right)
$$

The first term of Eq. (6) is called the computable correction. If the governing Eq. (1) is solved using a Galerkin approximation method, the computable correction contribution will vanish because the residual is orthogonal to all members of the finite element space. The second term in Eq. (6) is called the remaining error after the adjoint-based error correction and it cannot be evaluated because it requires the exact values of either $u$ or $v$.

Our next step is to develop an adaptive strategy that does not require the exact solutions, $u$ and/or $v$, of the direct and/or adjoint problems. Some authors $[5,9,10,13]$ advocate the use of the following strategy: since $u$ and $v$ cannot be computed with finite cost, one can approximate these on a finer grid with average spacing $h / 2$ :

$$
\epsilon_{h}=\left(v_{h}, R_{u}\right)-\left(v_{h}-v_{h / 2}, A\left(u_{h / 2}-u_{h}\right)\right) .
$$

Here, as indicated above, $R_{u} \equiv A u_{h}-f$. A computable form of the error estimate is derived by using a measure of the interpolation error in $u$ and $v$ that replaces $u_{h / 2}-u_{h}$ and $v_{h / 2}-v_{h}$, respectively, in the expression above. The interpolation error is expressed as the difference between the high- and low-order approximations to the primal and adjoint solutions on meshes of spacing $h / 2$, where the low-order approximation/interpolation $u_{h / 2}^{L}$ is usually obtained via piecewise linear interpolation and the high-order one, $u_{h / 2}^{H}$, is derived using quadratic interpolation obtained with Hermitian polynomials. The final form of the computable expression for the error estimator is then:

$$
\epsilon_{h} \approx\left(v_{h / 2}^{L}, R_{h / 2}\left(u_{h / 2}^{L}\right)\right)-\left(v_{h / 2}^{L}-v_{h / 2}^{H}, A_{h / 2}\left(u_{h / 2}^{H}-u_{h / 2}^{L}\right)\right)
$$

where $R_{h / 2}\left(u_{h / 2}^{L}\right)$ stands for the residual of the coarse grid solution on the fine grid.

It is important to note that, in this procedure:

1. The approximate flow solution is only required on the coarse grid. This key attribute avoids the cost of a flow solution on the finest grid.

2. The error estimator is evaluated on a grid that is finer $(h / 2)$ than the baseline grid $(h)$. But we are interested in the error of the solution evaluated in the baseline grid $(h)$ : Thus, a post- 
processing step is needed to obtain the error estimator in the baseline $(h)$ grid. In most of the cases, as the location of the coarse grid points coincide with some fine grid points, a simple injection is done to obtain the value of the adaptation sensor on the coarse grid.

These ideas allow the computation of an adaptation indicator without the knowledge of the exact solutions to the flow or adjoint problems. Such concepts have been tested by several authors $[5-7,9,13]$ with a significant level of success. Our intention in this article, however, is to develop a new approach that focuses instead on Uncertainty Quantification (UQ) applications. A robust numerical grid is defined as a computational grid in which the numerical error remains nearly constant for small variations of the parameters that affect the solution. In an ideal situation, the variation of the numerical error would be zero as a result of variations in the parameters of the problem.

For purposes of uncertainty quantification in our model problem, we assume that uncertainties enter the system through the right hand side $f$ of Eq. (1) and they are represented by the variation $\delta f$, which is unknown. This leads also to an unknown variation of the state $\delta u$ that can be computed using the following equation.

$$
\begin{cases}A \delta u=\delta f, & \text { in } \Omega \\ \delta u=0, & \text { on } \partial \Omega .\end{cases}
$$

Using Eq. (6), and keeping in mind that $v$ does not depend on $f$, it is possible to compute the variation of the numerical error as

$$
\delta \epsilon=\left(v_{h}, \delta R_{u}\right)-\left(v_{h}-v, A\left(\delta\left(u-u_{h}\right)\right)\right)
$$

where $\delta R_{u}=\delta\left(A u_{h}-f\right)=A \delta u_{h}-\delta f$. This estimation of the variation of the error $\delta \epsilon$ presents two main difficulties:

1. The exact solution of the adjoint problem, $v$, is required to evaluate the second term of the variation of the numerical error.

2. Eq. (10) requires the computation of variations of the residuals in Eq. (9). Note that, in principle, one adjoint problem should be solved for each uncertain parameter $\delta f$. 
To obtain an adaptation sensor, we apply an analogous strategy as the one used in Eq. (8). The variation of the error will be estimated in a finer grid and two interpolation operators (lowand high-order ones, respectively) will be used. In this way we obtain the following expression for the variation of the error

$$
\delta \epsilon_{h}=\left(v_{h / 2}^{L}, \delta R_{h / 2}\left(u_{h / 2}^{L}\right)\right)-\left(v_{h / 2}^{L}-v_{h / 2}^{H}, A\left(\delta\left(u_{h / 2}^{H}-u_{h / 2}^{L}\right)\right)\right)
$$

Note that a significantly-well refined grid for solving the primal problem $u_{h / 2}^{L}-u_{h / 2}^{H} \approx 0$ does not guarantee a low, or even a constant error variation. On the other hand, if a significantly-well refined grid is used to solve the adjoint problem, we expect that $v_{h / 2}^{L}-v_{h / 2}^{H} \approx 0$, and the variation of the error can be estimated as

$$
\delta \epsilon_{h} \approx\left(v_{h / 2}^{L}, \delta R_{h / 2}\left(u_{h / 2}^{L}\right)\right)
$$

Thus, in order to estimate (in order to later reduce) the value of $\delta \epsilon_{h}$ it is necessary to evaluate $\delta R_{h / 2}\left(u_{h / 2}^{L}\right)$ which, itself, requires the solution of the sensitivity problem in Eq. (9). In the next section we introduce the way in which this quantity is used on practical grid adaptation procedures.

\section{B. Practical implementation}

The robust adaptation technique seeks a grid with low numerical error even under variations of the input parameters of the governing equation. Such a grid would require that:

$$
\left\{\begin{array}{l}
v_{h / 2}^{L}-v_{h / 2}^{H} \approx 0 \\
v_{h / 2}^{L} \delta R_{h / 2}\left(u_{h / 2}^{L}\right) \approx 0
\end{array}\right.
$$

where, in order to satisfy the first equation, we can adapt the numerical grid using as indicator the value of $R_{h / 2}\left(v_{h / 2}^{L}\right)$. On the other hand, note that the second equation arises from the estimation of the error variation. It is important to note that, in order to obtain a well-resolved adjoint solution, one could also propose an a posteriori error estimation procedure for the adjoint variables, that would result in an adaptation method based on a weighted residuals. As before, note that $R_{h / 2}$ represents the residual on the finest grid and $u_{h / 2}^{L}$ and $v_{h / 2}^{L}$ are the flow and adjoint solutions (respectively) computed on the coarse grid and interpolated to the finest grid using a low-order (linear) interpolator. To simplify the notation, $\delta R^{U}$ and $R^{V}$ will be used to represent $\delta R_{h / 2}\left(u_{h / 2}^{L}\right)$ 
and $R_{h / 2}\left(v_{h / 2}^{L}\right)$, respectively. Also, the low-order approximation of the adjoint solution $v_{h / 2}^{L}$ will be denoted by $v$. To achieve a solution with a low numerical error of the adjoint solution on the baseline grid (first equation of Eq. (13)), we require that $R^{V} \approx 0$. Moreover, since the adjoint operator - in a general framework - will depend on the flow solution (e.g. for Euler equations the adjoint operator is the transpose of the Jacobian of the fluxes) it is also desirable to have $R^{U} \approx 0$. As a result, the numerical grid must be adapted in order to minimize the following objective function:

$$
e_{\text {res }}=\left\|R^{U}\right\|^{2}+\left\|R^{V}\right\|^{2}
$$

As far as the robust computable correction terms are concerned and if we assume that we have $N$ uncertain parameters that change the values of the right hand side, $f$, in Eq. (1), the objective function to be minimized through the adaptation process is

$$
e_{\text {comp }}=\sum_{i=0}^{N}\left\|v \delta R_{i}^{U}\right\|^{2},
$$

where $\delta R_{i}^{U}$ is the sensitivity of the primal problem residual to variations in the $\mathrm{i}$-th uncertain parameter.

In summary, using the robust grid strategy it is possible to define two different error indicators: the Residual Robust Adaptation (RRA) error indicator, Eq. (14), and the Computable Robust Adaptation (CRA) error indicator, Eq. (15). Note that the RRA method will adapt a numerical grid to minimize the error in the adjoint solution. In this way, the variation of the numerical error will depend only on the residual of the linearized solution, scaled by the adjoint variables.

The final form of a global error indicator is then:

$$
e_{\text {ind }}=e_{\text {res }}+e_{\text {comp }}
$$

Note that, when dealing with non-linear problems, it is customary to multiply the error in each computational cell by $|\Omega|^{\beta}$ where $|\Omega|$ is the volume of a particular cell. The advantage of scaling the indicator with a positive value of $\beta$ is that the adaptation can terminate automatically in relevant regions after several cycles, even when discontinuities are present in the flow field.

From a practical point of view, it is useful to define a limit for the grid adaptation process so that a stopping criterion exists:

$$
e_{l i m}=e_{m}+c_{l i m} e_{s}
$$


where $e_{\text {lim }}$ is the error limit, $e_{m}$ is the mean of the error indicator, $e_{s}$ is the standard deviation of the error indicator, and $c_{l i m}$ is a user-specified constant. Typically, a value near unity is used for this constant. More detailed information on the implementation of error limits for grid adaptation can be found in Ref. [6]. Finally, to carry out the refinement process, we use a standard homothetic subdivision approach [14], that splits each triangle into four new triangles by adding three new vertices at the middle of each edge. It has to be noted that elements sharing these edges must also be divided if hanging nodes are to be avoided.

\section{Numerical examples}

The objective of this section is to illustrate the performance of the new adaptation techniques described in this article:

1. The RRA method based on the use of a function of the direct and adjoint residuals as an adaptation sensor, see Eq. (14). In a ideal scenario this kind of adaptation will remove the influence of the second term that appears in the error variation, see Eq. (11).

2. The CRA method based on the use of the adjoint problem multiplied by the residual of the linearized problem as adaptation sensor, see Eq. (15).

In addition, in this section we also compare these new adaptation techniques with three well know non-robust adaptation methodologies:

1. Uniform adaptation based on the uniform subdivision of each cell of the mesh.

2. Computable correction based on an adaptation sensor constructed with the residual of the direct problem multiplied by the adjoint solution, see Eq. (8).

3. Gradient-based adaptation where the flow field is adapted in areas where the gradient of the direct solution is greater than a prescribed value.

It is important to highlight that, once the adaptation sensor is computed, the rest of the adaptation methodology is the same for all the methods. 


\section{A. 2D Poisson's equation on structured grids}

In order to clarify the robust adaptation method described above, we will solve the twodimensional Poisson equation based on an example proposed by Pierce and Giles in Ref. [15]

$$
\begin{cases}\nabla^{2} u=x(1-x)^{\alpha} y(1-y), & \text { in } \Omega, \\ u=0, & \text { on } \partial \Omega\end{cases}
$$

on the unit square $[0,1] \times[0,1]$ subject to homogeneous Dirichlet boundary conditions, and where $\alpha$ is an uncertain parameter. We also consider the following objective function:

$$
\int_{\Omega} u \sin (\pi x) \sin (\pi y) d V
$$

The dual or adjoint problem is given by

$$
\begin{cases}\nabla^{2} v=\sin (\pi x) \sin (\pi y), & \text { in } \Omega \\ v=0, & \text { on } \partial \Omega\end{cases}
$$

The equation for the sensitivity of the solution with respect to the source term is

$$
\begin{cases}\nabla^{2}(\delta u)=x(1-x)^{\alpha} y(1-y) \ln (1-x) \delta \alpha, & \text { in } \Omega \\ \delta u=0, & \text { on } \partial \Omega .\end{cases}
$$

These equations are approximated using a classical Galerkin Finite Element Method with piecewise linear elements on a numerical grid made up of triangles. Fig. 1 shows the numerical solution of the direct problem for $\alpha=1$. (left), the adjoint solution (middle), and the solution of the sensitivity problem (right) for $\delta \alpha=1$. The baseline triangulation was constructed by splitting each quadrilateral element of a Cartesian mesh into two triangles.

In order to estimate the exact solution, a very fine grid with 10,000 nodes is used. As a baseline for the adaptation process, we use a numerical grid with only 1,600 nodes. The numerical experiment involves computing the value of the functional for $1.0<\alpha<2.0$ and comparing the numerical error for the following adaptation strategies: 1) Computable method $\left.\min \left(v, R^{U}\right), 2\right) \mathrm{CRA}$ $\operatorname{method} \min \left(v, \delta R^{U}\right)$, and 3) RRA method $\min R^{U}$ and $\min R^{V}$.

In Fig. 2 (left), the value of the functional on the finest grid is plotted for different values of $\alpha$. In Fig. 2 (right) the difference (error) between the truth solution and the numerical solution on the 

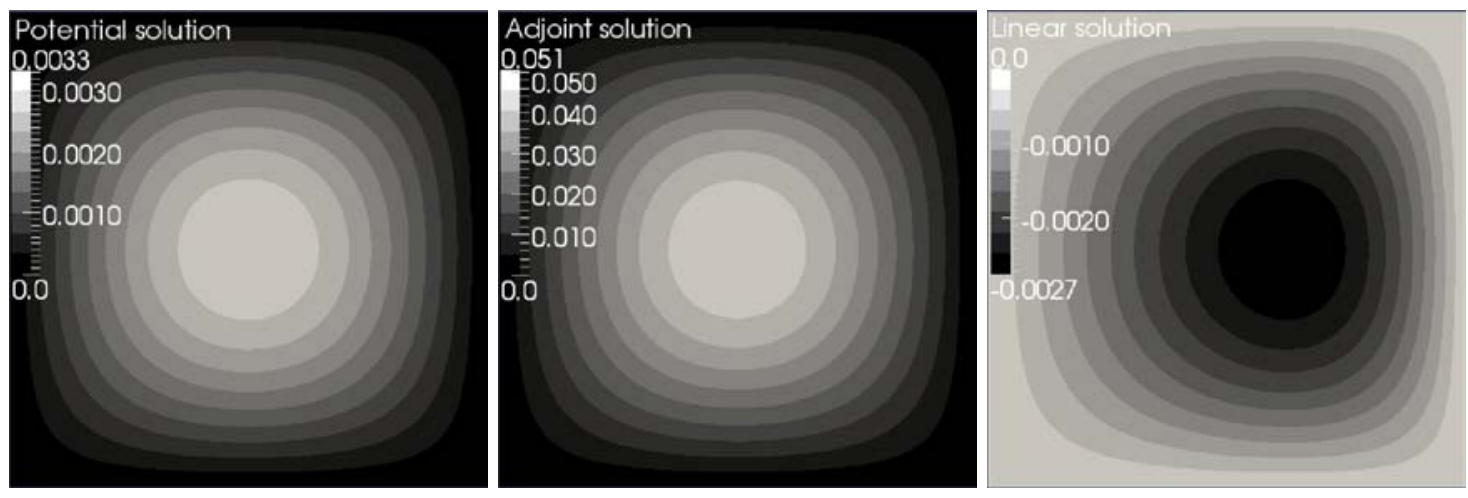

Fig. 1 Solution of the direct (left), adjoint (middle) and sensitivity (right) problems on the finest grid (10.000 nodes), for $\delta \alpha=1.0$.
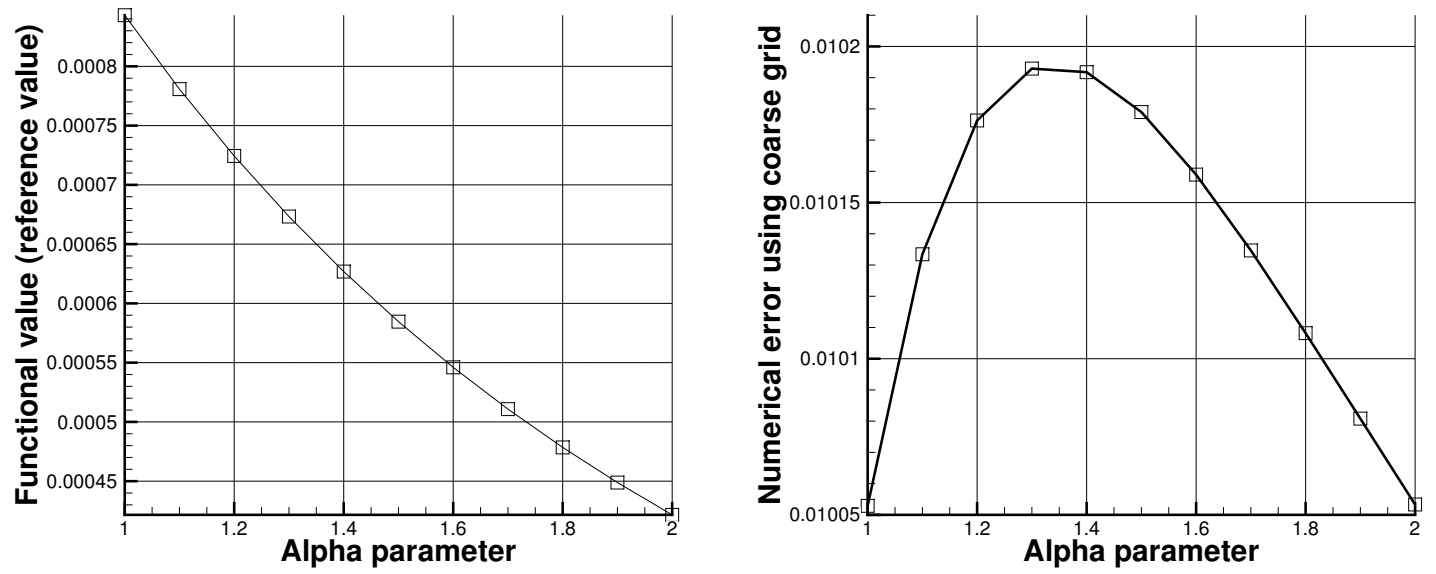

Fig. 2 Functional value for different values of the parameter $\alpha$ (left) and numerical error in the 1600 nodes grid (right) for different values of $\alpha$.
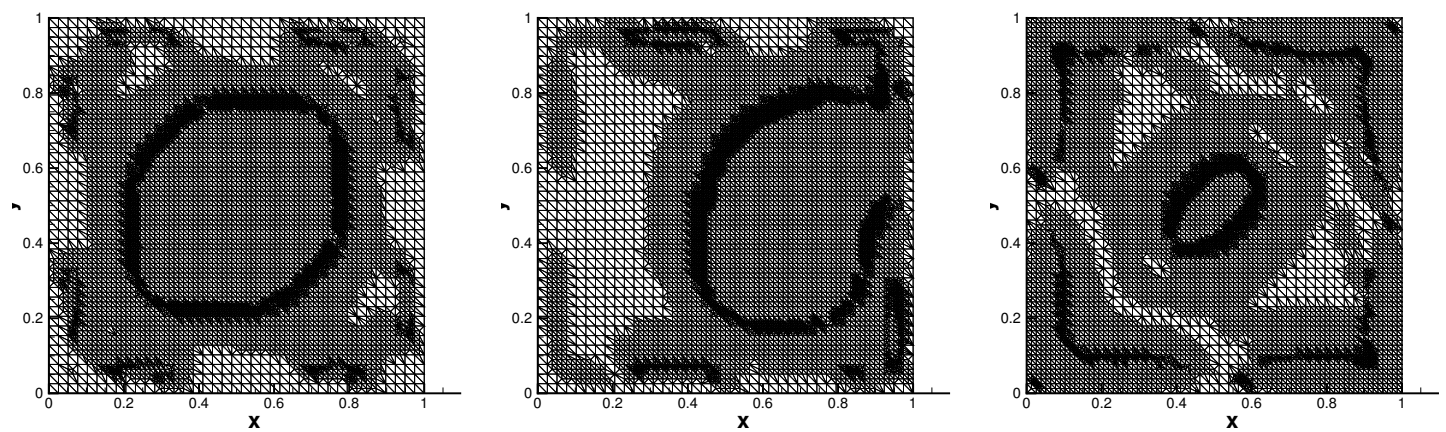

Fig. 3 Numerical grid after adaptation using computable adaptation (left), CRA method (middle), and RRA method (right). 

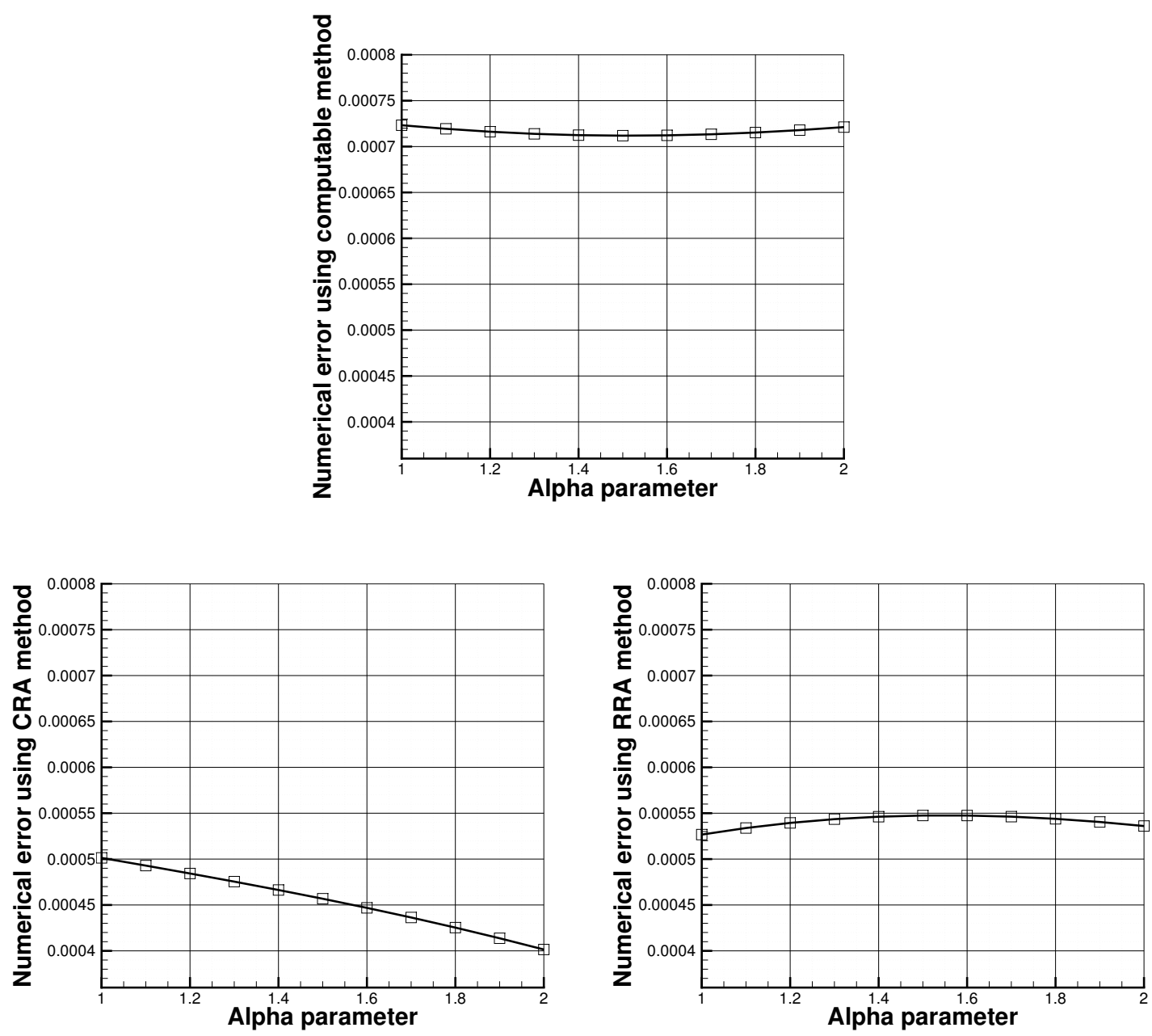

Fig. 4 Numerical error using computable (upper), CRA (lower left) and RRA (lower right) methodology.

coarse grid is shown. In Fig. 3, the resulting grids using the computable error (left), CRA method (middle), and RRA methods (right) are shown. The number of points of the computational grids are approximately the same: $6,806,6,721$, and 6,862 , respectively.

Finally, in Fig. 4, a comparison between the numerical errors for different values of $\alpha$ is shown. In all cases the numerical error is under $1 \%$, but the CRA method produces a lower error level than the others. Regarding the shape of distribution of the error, it is important to highlight that the RRA method maintains the same behavior as the original coarse grid. However, using the CRA method, the error derivative with respect to the uncertain parameter is constant. Moreover, the computational grid adapted using the Computable Error method (one point adaptation) shows a 
minimum for the error at $\alpha=1.5$, pointing to the fact that the grid has been adapted to a specific value of the parameter, but ,due to imprecisions of the adaptation procedure, the minimum error is not exactly at $\alpha=1.0$, as we could have expected.

\section{B. Application of CRA method to variations in free stream Mach number using the Euler equations}

Inviscid compressible flows are governed by the Euler's equations, which express mathematically the statements of conservation of mass, momentum (with null viscosity), and energy. The conservative steady-state form of the 2D Euler equations is given by:

$$
\left\{\begin{array}{l}
\vec{\nabla} \cdot \vec{F}=0, \\
\vec{v} \cdot \vec{n}=0, \quad \text { in } \Omega,
\end{array}\right.
$$

where $\vec{n}$ is an inward-pointing unit vector normal to $S$ (solid surface). At the far-field, boundary conditions are specified for incoming waves, whereas outgoing waves are determined by the solution inside the fluid domain.

In two dimensions, the conservative variables are $U=\left(\rho, \rho v_{x}, \rho v_{y}, \rho E\right)^{T}$ and $\vec{F}=\left(F_{x}, F_{y}\right)$ is the convective flux vector

$$
F_{x}=\left(\begin{array}{c}
\rho v_{x} \\
\rho v_{x}^{2}+P \\
\rho v_{x} v_{y} \\
\rho v_{x} H
\end{array}\right), \quad F_{y}=\left(\begin{array}{c}
\rho v_{y} \\
\rho v_{x} v_{y} \\
\rho v_{y}^{2}+P \\
\rho v_{y} H
\end{array}\right),
$$

where $\rho$ is the fluid density, $\vec{v}=\left(v_{x}, v_{y}\right)$ is the flow velocity vector in a Cartesian reference frame, $E$ is the total energy of the fluid (internal plus kinetic), $P$ the static pressure, and $H$ the enthalpy. The system of equations (Eq. (22)) must be completed by an equation of state which defines the thermodynamic properties of the fluid. In this case, we use the ideal gas law $p=\rho R T$.

The linearized Euler's equations (for smooth solutions of the direct problem) with respect to variations on the shape of the solid surface $S$ or in the far-field are:

$$
\begin{cases}\vec{\nabla} \cdot\left(\frac{\partial \vec{F}}{\partial U} \delta U\right)=0, & \text { in } \Omega, \\ \delta \vec{v} \cdot \vec{n}=-\delta S \partial_{n} \vec{v} \cdot \vec{n}+\left(\partial_{t} \delta S\right) \vec{v} \cdot \vec{t} & \text { on } S, \\ (\delta W)_{+}=0, & \text { on } \Gamma_{\infty},\end{cases}
$$


where the variable $\delta U$ solves the linearized Euler's equations, $\partial_{n}=\vec{n} \cdot \vec{\nabla}$ and $\partial_{t}=\vec{t} \cdot \vec{\nabla}$ are the normal and tangential derivative operators (respectively), and $(\delta W)_{+}$represents the incoming characteristics in the far-field. More details of the notation used for the linearized problem can be found in [16].

On the other hand, the continuous adjoint system for Euler's equations (again, for smooth solutions of the direct problem) is given by:

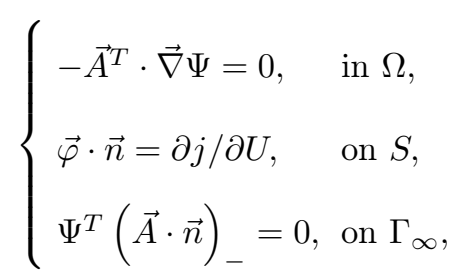

where $\left(\Psi^{T}\right)=\left(\psi_{1}, \psi_{2}, \psi_{3}, \psi_{4}\right)$ are the adjoint variables, $j$ is the functional of interest, the Jacobian is written as $\partial \vec{F} / \partial U=\vec{A}$, and $\vec{\varphi}=\left(\psi_{2}, \psi_{3}\right)$. As before, a characteristic-based method is used at the far-field boundary. Again, more details of the notation used for the adjoint system and its development can be found in [17].

For this example, a NACA0012 airfoil in the subsonic regime (Mach number 0.5 and angle of attack 2.0) has been chosen as the baseline configuration. The objective of this numerical test is to compare the performance of the CRA strategy (for variations in the freestream Mach number from 0.4 to 0.6 ) with more classical computable adaptation procedures. The objective function throughout this example is taken to be the lift coefficient of the airfoil. As a starting point, a baseline grid consisting of 1,341 nodes (see Fig. 5, left) is created. Then, three homothetic isotropic grid adaptation cycles were performed and, in Fig. 6 (left), the value of the airfoil lift coefficient is plotted for the different grids. Note that an analytical definition of the airfoil surface is used to add new nodes over the surface to guarantee numerical convergence.

The next step is to adapt the baseline grid using the new computable robust adaptation (CRA) strategy, and a classical computable adaptation approach [9]. In Fig. 5 (middle) the numerical grid adapted with the computable approach is presented, and in Fig. 5 (right) the new robust adapted grid is shown (using CRA). Note that we have performed 2 adaptation cycles and the final number of points is 3,097 (for the classical computable approach), and 3,075 (using the CRA strategy).

In order to evaluate the errors in the numerical results, we have computed a solution on a 

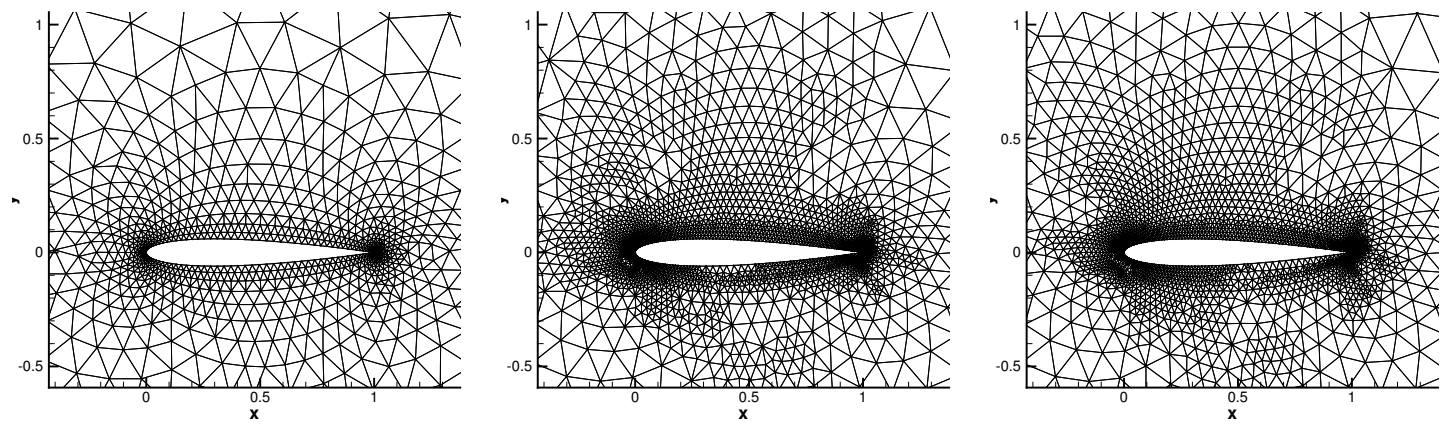

Fig. 5 Coarse/baseline grid (left), numerical grid obtained with the computable adaptation strategy (middle), and numerical grid obtained with the CRA strategy (right).
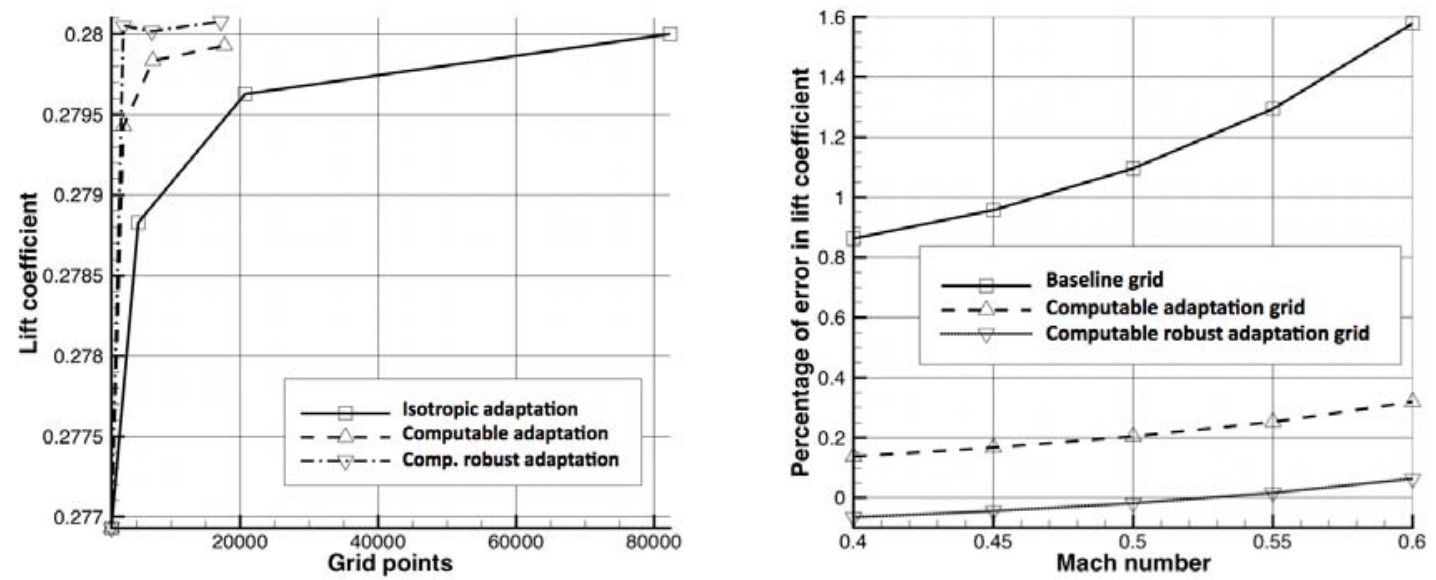

Fig. 6 Convergence history for different adaptation strategies (left), and lift coefficient value for different Mach numbers (right).

uniformly-refined grid containing 82,324 points that will be referred to as the truth solution. We have then computed the error in the lift coefficient using the baseline/isotropic, computable, and CRA procedures with respect to this solution. Note that, while the baseline Mach number had been selected as 0.5, results have been computed for different values of the free stream Mach number, note that the adaptation criteria were computed using the adjoint solution corresponding to the baseline Mach number 0.5. In Fig. 6 (right), the error in the lift coefficient is plotted for the three adaptation approaches as a function of the free stream Mach number.

One can see from the results in Fig. 6 that, just as in the Poisson equation example, the CRA 
strategy not only results in a more robust grid (in the sense that it is less sensitive to variations in the freestream Mach number), but also provides for smaller numerical errors with essentially the same number of points than the classical computable adaptation. It is important to highlight that in both adjoint-based adaptation strategies, the solution of the adjoint system of equations is necessary and, therefore, the differences between the results for the classical computable strategy and the CRA approach represent true differences for a given computational budget. The isotropic/baseline adaptation strategy does not require the solution of an adjoint system and is therefore computed at a lower cost than the other two approaches. However, observing Fig. 6 (left), just doubling the points of the isotropic adaptation strategy (to obtain an equivalent computational effort as solving the adjoint problem) is not sufficient to obtain a competitive lift error with respect to the other strategies.

\section{Response surface computation for supersonic inlet flows using the RRA method}

Uncertainty quantification using non-intrusive methods such as stochastic collocation typically involves the construction of a response surface of the quantity of interest as a function of the variation of several uncertain parameters. To achieve the desired level of accuracy, a large number of CFD simulations is typically required. To improve the efficiency of this process, we propose to employ our robust grid adaptation technique that is aimed at minimizing the numerical error over small variations of the parameters around a nominal state.

To study the applicability of this method, a two-dimensional Euler supersonic inlet test case has been selected. In particular, we are interested in creating a robust grid for a subset of the Clemens experimental configuration [18]. The nominal conditions (as in the experiments) are an inlet Mach number of 5.0 and a ramp angle 6.0 (Fig. 7). The adjoint computation (Fig. 8) has been performed using a second-order continuous adjoint formulation. The objective of this test is to verify whether the grid adapted (using the new robust technique) for those nominal conditions also provides a good value of the objective function for the complete response surface. In this case we are interested in predicting accurately the pressure signature in an area of the lower wall that is located slightly downstream of the end of the inlet ramp. This choice is made as a surrogate for various 
Fig. 7 Density contours for nominal conditions (inflow Mach number 5.0 and ramp angle $6.0^{\circ}$ )

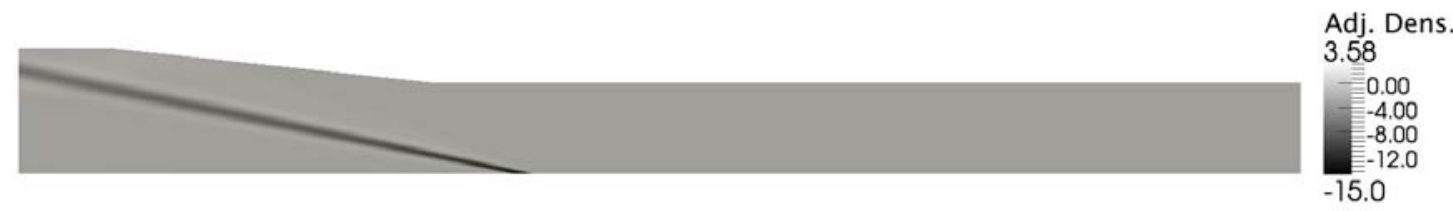

Fig. 8 Adjoint density contours for nominal conditions and pressure sensor located on the lower wall
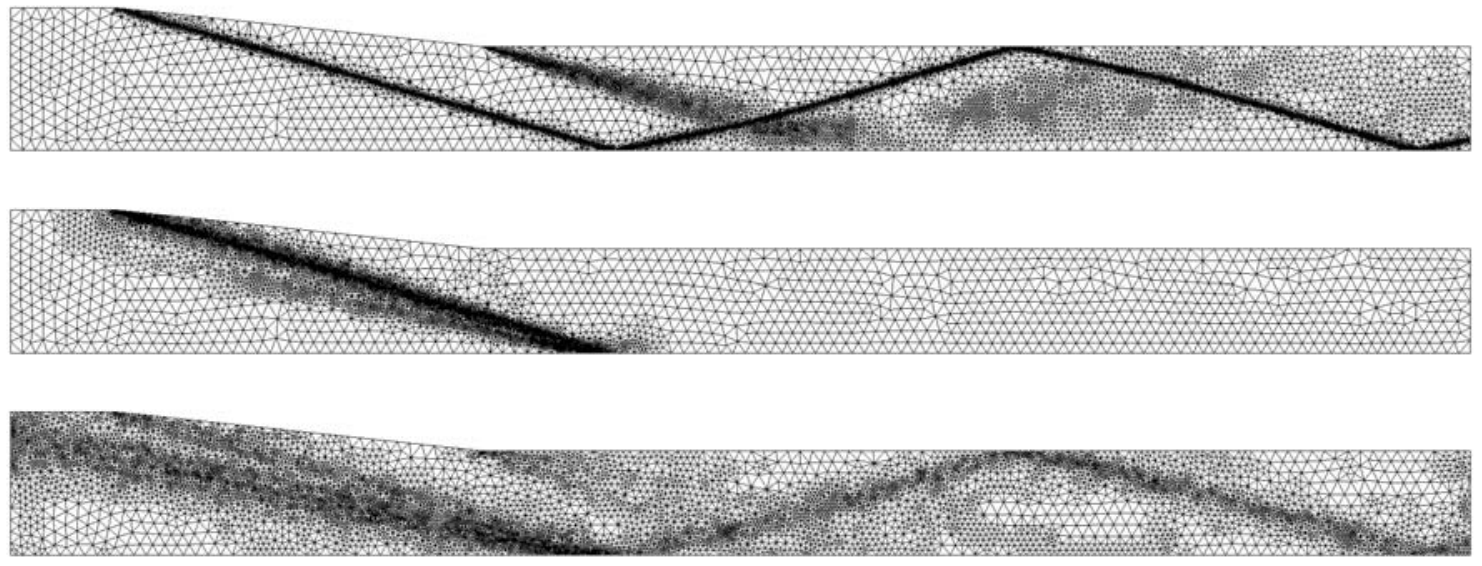

Fig. 9 Grid adaptation using a gradient-based method (upper), adjoint-based computable error (middle), and the new robust method (lower)

measures of precursors to the unstart phenomenon that we are exploring in other current efforts [19]. The baseline grid has a total of 1,356 nodes and 2, 450 triangular elements. The total length of the setup is $333.0 \mathrm{~mm}$, and the pressure sensor is located on the lower wall at $116.08 \mathrm{~mm}$ from the inlet (the size of the pressure sensor is $5.08 \mathrm{~mm}$ ). In order to compare the performance of the different grid adaptation techniques (Fig. 9) we have used three different error indicators: gradient based, classical adjoint based (computable error), and the new RRA method. It is important to 

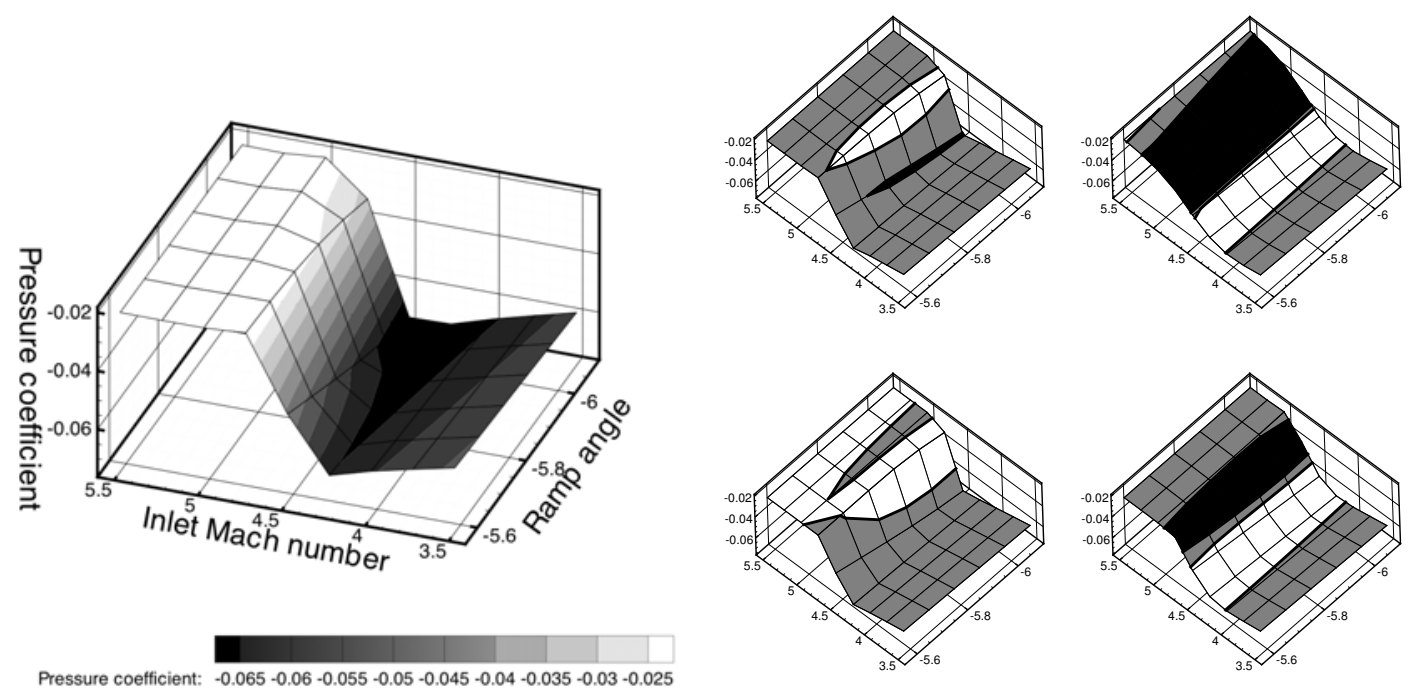

Fig. 10 Fine grid solution (baseline response Fig. 11 Quantitative comparison between difsurface)

\author{
ferent adaptation techniques and the fine grid \\ solution: RRA method (upper left), no adap- \\ tation (upper right), computable error (lower \\ left), and gradient based adaptation (lower \\ right). In all cases gray shading represents \\ an error $<4.0 \%$
}

note that, even though the flow conditions are uniform at the inlet, the RRA method indicates a need for adaptation along certain portions of the inlet. This is because the RRA method is based on minimizing the flow and adjoint residuals and the adjoint residuals will be active at the inlet due to the non-uniformity of the adjoint variables.

Once the adaptation (based on the nominal conditions) is complete, the final step consists of computing the entire response surface of the quantity measured by the pressure sensor (Fig. 11) and comparing the results with the "exact" response surface that has been computed using a very fine reference grid. In this particular simulation, the Mach number varies from 3.5 to 5.5, while the ramp angle changes from 5.6 to 6.1 degrees (see Fig. 10).

In Fig. 11, we compare four different methods for grid adaptation with the solution obtained on a very fine grid. The grey color represents an absolute value of the error that is less than $4.0 \%$. Starting with the no adaptation case, the numerical error is greater than $4.0 \%$ almost everywhere 


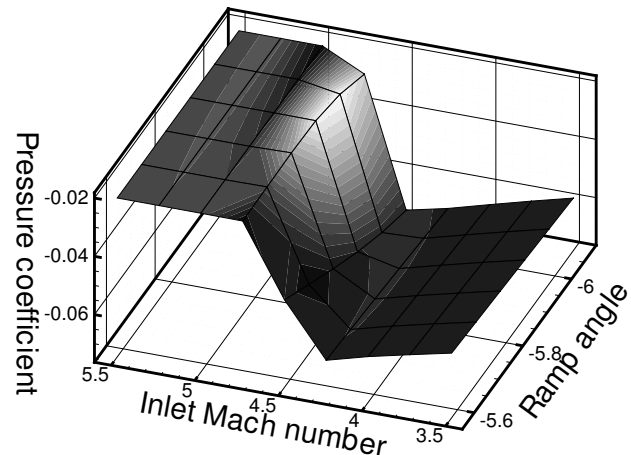

Error comp.: $0 \begin{array}{lllllllllllllll} & 1 & 2 & 3 & 4 & 5 & 6 & 7 & 8 & 9 & 1011121314151617\end{array}$

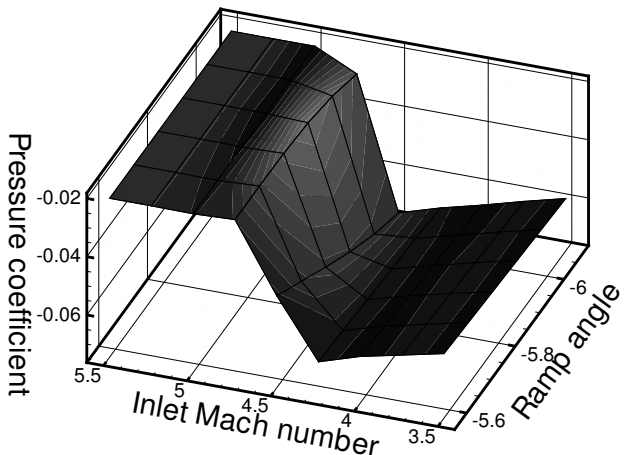

Error RRA: $\begin{array}{lllllllllll}0 & 1 & 2 & 3 & 4 & 5 & 6 & 7 & 8 & 9 & 1011121314151617\end{array}$

Fig. 12 Direct comparison between computable error based adaptation (left) and RRA methodology (right)

except when the shock wave is far from the pressure sensor (for the entire Mach number range). For the flow gradient adaptation, a similar behavior is observed, but the zones with error greater than $4.0 \%$ are narrower. Using the computational correction adaptation, it is interesting to note that the error is lower than $4.0 \%$ at the baseline design point, but increases rapidly with changing Mach number. As in other cases, however, when the shock is far from the sensor, the numerical error is low. Finally, as expected, the robust grid adaptation method produces the best results: the numerical error is lower than in the other cases except when the shock wave crosses the pressure sensor at Mach number 4.75. This particular flow condition is, however, difficult for all methods due to the presence of strong pressure gradients.

In conclusion, for the complete response surface computation, the robust grid strategy provides a lower error compared with the other techniques, despite the fact that only one adaptation around Mach 5.0 and ramp angle $6.0^{\circ}$ is used. In particular, using the computational correction adaptation method we obtain a numerical error less than $4.0 \%$ in approximately $70 \%$ of the response surface, while using the RRA method, $90 \%$ of the flow domain has a numerical error less than $4.0 \%$.

It is important to note that, since adjoints are used, the proposed adaptation strategy provides an indication of regions that the functional of interest is most sensitive to. Even though different 
grids are employed for changes in geometric parameters (ramp angle in this case), regions of refinement are based on adaptation indicators developed around the baseline state and are held fixed while parameters are varied.

\section{Conclusions}

In this paper, we have described the foundations of two new grid adaptation strategies termed the Computable Robust Adaptation method (CRA) and the Residual Robust Adaptation method (RRA). We have shown that it is possible to obtain a grid that is robust with respect to small variations of the input parameters (in the sense that the numerical error is constant even if some input parameters are changed). This technique can be useful in improving the efficiency and accuracy in applications such as optimal shape design, response surface development, robust design/optimization, and in uncertainty quantification of systems governed by partial differential equations, the initial motivation of this work. Based on preliminary results on model problems, we have also shown that numerical grids adapted using the CRA method exhibit better properties than those adapted using a classical computable adaptation method for goal-oriented error estimation and adaptation. The main drawback of this new CRA technique is the requirement of solving an additional linearized problem for each variable of the problem, for that reason we have developed the RRA method, which is based on adapting independently the direct and adjoint residuals. With regard to computational cost, the RRA method is equivalent to two CFD runs on the coarse grid, while, on the other hand, the CRA method requires $N+2$ runs, where $N$ is the number of uncertain parameters.

This technique has been tested in a response surface computation and has demonstrated a

numerical error that is less than $4.0 \%$ in $90.0 \%$ of the domain of the response surface, while a computational correction-based adaptation produced acceptable results in only $70.0 \%$ of the response surface.

It has to be emphasized that the proposed approach has been developed for use in problems with small perturbations of input variables from a baseline state. The error estimate is not guaranteed to be accurate for large variations of the parameters since the adjoint solution is evaluated only for one combination of parameters. If utilized in uncertainty quantification with large parameter 
variations, adjoint solutions may need to be evaluated around multiple sample points and grid adaptation indicators may be derived using a composite of these samples.

Future work will be focused on improving this new technique when applied to non-linear equations, and to seek clear explanation as to why the CRA results in lower numerical errors than the classical computable method even at the design point.

\section{Acknowledgments}

This work was supported by the U.S. Department of Energy under the Predictive Science Academic Alliance Program (PSAAP). The third author was partially supported by Grant MTM200803541 and MTM2011-29306, MICINN, Spain, ERC Advanced Grant FP7-246775 NUMERIWAVES, ESF Research Networking Programme OPTPDE and Grant PI2010-04 of the Basque Government.

\section{References}

[1] Ghanem, R., "Hybrid stochastic finite elements and generalized Monte Carlo simulation," Journal of Applied Mechanics - ASME, Vol. 65, 1998, pp. 1004-1009.

[2] Hosder, S., Walters, R., and Perez, R., "A nonintrusive polynomial chaos method for uncertainty propagation in CFD simulations," AIAA Meeting Paper 2006-0891, 2006.

[3] Najm, H. N., "Uncertainty Quantification and Polynomial Chaos Techniques in Computational Fluid Dynamics," Annual Review of Fluid Mechanics, Vol. 41, 2009, pp. 35-52.

[4] Pierce, N. A. and Giles, M. B., "Adjoint and defect error bounding and correction for functional estimates," Journal of Computational Physics, Vol. 200, 2002, pp. 769-794.

[5] Park, M. A., "Three-dimensional turbulent RANS adjoint based error correction," AIAA Meeting Paper 2003-3849, 2003.

[6] Fidkowski, K. J. and Darmofal, D. L., "Review of Output-Based Error Estimation and Mesh Adaptation âĂlin Computational Fluid Dynamics," Tech. rep., April 2011.

[7] Giles, M. B. and Pierce, N. A., "Adjoint error correction for integral outputs," Tech. Rep. 01/18, Oxford University Computing Laboratory, Numerical Analysis Group, Oxford, England., 2001.

[8] Duraisamy, K., Alonso, J. J., Palacios, F., and Chandrashekar, P., "Error Estimation for High Speed Flows Using Continuous and Discrete Adjoints," AIAA Meeting Paper 2010-128, 2010.

[9] Venditti, D. A. and Darmofal, D. L., "Anisotropic Grid Adaptation for Functional Outputs: âĂÍApplication to Two-Dimensional Viscous Flows," Journal of Computational Physics, Vol. 187, 2003, pp. 22-46. 
[10] Nemec, M., Aftosmis, M. J., and Wintzer, M., “Adjoint-based Adaptive Mesh Refinement for Complex Geometries," AIAA meeting Paper 2008-0725, 2008.

[11] Eldred, M. and Burkardt, J., "Comparison of Non-Intrusive Polynomial Chaos and Stochastic Collocation Methods for Uncertainty Quantification," AIAA Meeting Paper 2009-0976, 2009.

[12] Giles, M. B., Pierce, N. A., and Süli, E., "Progress in adjoint error correction for integral functionals," Computing and Visualization in Science, Vol. 6, 2004, pp. 113-121.

[13] Kim, H.-J. and Nakahashi, K., "Output-Based Error Estimation and Adaptive Mesh Refinement Using Viscous Adjoint Method," AIAA Meeting Paper 2006-1395, 2006.

[14] Borouchaki, H., Villard, J., Laug, P., and George, P. L., "Surface mesh enhancement with geometric singularities identification," Computer Methods in Applied Mechanics and Engineering, Vol. 194, 2005, pp. $4885-4894$.

[15] Giles, N. A. P. M. B., "Adjoint recovery of superconvergent functionals from approximate solutions of partial differential equations," Oxford university computing laboratory report na 98/18, 1998.

[16] Baeza, A., Castro, C., Palacios, F., and Zuazua, E., "2-D Euler shape design on nonregular flows using adjoint Rankine-Hugoniot relations," AIAA Journal, Vol. 47, No. 3, 2009, pp. 552-562.

[17] Bueno-Orovio, A., Castro, C., Palacios, F., and Zuazua, E., "Continuous adjoint approach for the Spalart-Allmaras model in aerodynamic optimization," AIAA Meeting Paper 2011-1299, 2011.

[18] Wagner, J. L., Valdivia, A., Yuceil, K. B., Clemens, N. T., and Dolling, D. S., "An experimental investigation of supersonic inlet unstart," AIAA Meeting Paper 2007-4352, 2007.

[19] Wang, Q., Duraisamy, K., Alonso, J., and Iaccarino, G., "Risk Assessment of Hypersonic Flow Simulations Using Adjoint-Based Sampling Methods," AIAA Meeting Paper 2010-2921, 2010. 